\title{
Acute onset of severe livedo reticularis in a patient with myocardial infarction
}

\author{
Fabiola Schafer ${ }^{1}$, Rodrigo Binder ${ }^{2}$, Enrique Bellolio ${ }^{3}$, Rodrigo Miranda ${ }^{4}$ \\ 1Department of Medical Specialties, School of Medicine, Universidad de La Frontera, Temuco, Chile \\ ${ }^{2}$ Cardiology Unit, Doctor Victor Rios Ruiz Medical Complex Hospital, Los Angeles, Chile \\ ${ }^{3}$ Department of Anatomic Pathology, School of Medicine, Universidad de La Frontera, Scentific and Technological Bioresource Nucleus \\ (BIOREN),Temuco, Chile \\ ${ }^{4}$ Department of Internal Medicine, School of Medicine, Universidad de La Frontera, Temuco, Chile
}

Adv Dermatol Allergol

DOI: https://doi.org/10.5114/ada.2020.100820

Livedo reticularis is a cutaneous sign that presents with red violaceous patches with a net-like pattern and mainly affects the lower extremities. It is produced by an interruption of blood flow in the dermal arteries, due to spasm, inflammation or vascular obstruction. So, it can be associated with different aetiologies.

We present a case of severe livedo reticularis in a patient with acute myocardial infarction.

A 49-year-old man, active smoker, was admitted with an inferior ST elevation myocardial infarction and then thrombolysis with streptokinase was done with successfully reperfusion criteria. At night, the patient developed fever, myalgia, hypotension, tachycardia and painful red violaceous patches in a net-like pattern on his lower extremities associated with ulcers, blisters and purple discoloration of the toes and soles of both feet, which rapidly extended to pelvic area (Figures 1 A, B). The distal pulses were preserved. During the first days, a progressive rhabdomyolysis and renal failure were detected, in fact, the blood test showed creatinine phosphokinase of $12,000 \mathrm{mg} / \mathrm{dl}$ and creatinine of $4.5 \mathrm{mg} / \mathrm{dl}$ associated with mild eosinophilia of 672 cells/ $\mu$. Thorax-abdomen computed tomography (CT) scan with contrast showed ulcerated atherothrombosis of the abdominal aorta (Figure $1 \mathrm{C}$ ). Therefore, lower extremities embolization syndrome was suspected.

The immunological panel was negative and sepsis was ruled out. A skin biopsy showed dermoepidermal necrosis and arteriolar vessel microthrombosis with no signs of vasculitis or inflammatory cell infiltration, compatible with atheroembolism (Figures 1 D, E). The patient presented with acute distress syndrome and renal failure requiring mechanical ventilation and haemodialysis in an intensive care unit.

After 3 weeks of supportive treatment, the patient recovered his renal function and the skin lesions slowly improved with medical wound care, however, amputation was required of his fifth right toe and part of his left hallux due to gangrene. A long-term follow-up did not show any other organic affectation.

Atheroembolism is a very rare complication of thrombolytic therapy. It produces a microembolism (thrombose or cholesterol crystal) from ulcerated atherosclerotic plaques to any organ and the circulation of the distal lower limbs, causing occlusion of dermal arterioles, ischaemic infarction of the epidermis, desaturation of blood and cyanosis in the toes [1]. Interestingly, the kidney and skin are the most commonly affected organs. Renal failure and cutaneous manifestations such as livedo reticularis, blue toe syndrome, ulcers, purpura and digital gangrene are common signs [2]. Skin lesions can be the only clinical presentation, although embolization syndrome has the potential to cause multi-organ involvement of the brain, eyes, gastrointestinal tract and kidneys. Its mortality rate is up to 70-90\% [3]. The diagnosis is suspected by clinical presentation and confirmed by the biopsy which may be made from the skin, kidney or muscle [4]. In our case, the diagnosis was suspected by the presence of livedo reticularis, blue toe syndrome, renal failure and eosinophilia.

Other treatments such as angiographic procedures, vascular surgery or anticoagulation therapy (warfarin) have also been described to produce embolization syndrome [5]. Usually, patients have unstable atherosclerotic lesions over the entire aorta. Male gender, advanced age, acute coronary syndrome, multivessel coronary artery disease, hypertension, smoking, cerebrovascular disease and aortic aneurysm have been recognized as major risk factors for embolization syndrome [2]. In our patient, risk factors such as male gender, acute coronary syndrome and active smoking were present.

Address for correspondence: Fabiola Schafer MD, Department of Medical Specialties, School of Medicine, Universidad de La Frontera, 115 Claro solar St, Temuco, Chile, phone: 56-45-744322, e-mail: fdschafe@gmail.com Received: 19.10.2020, accepted: 29.10.2020. 

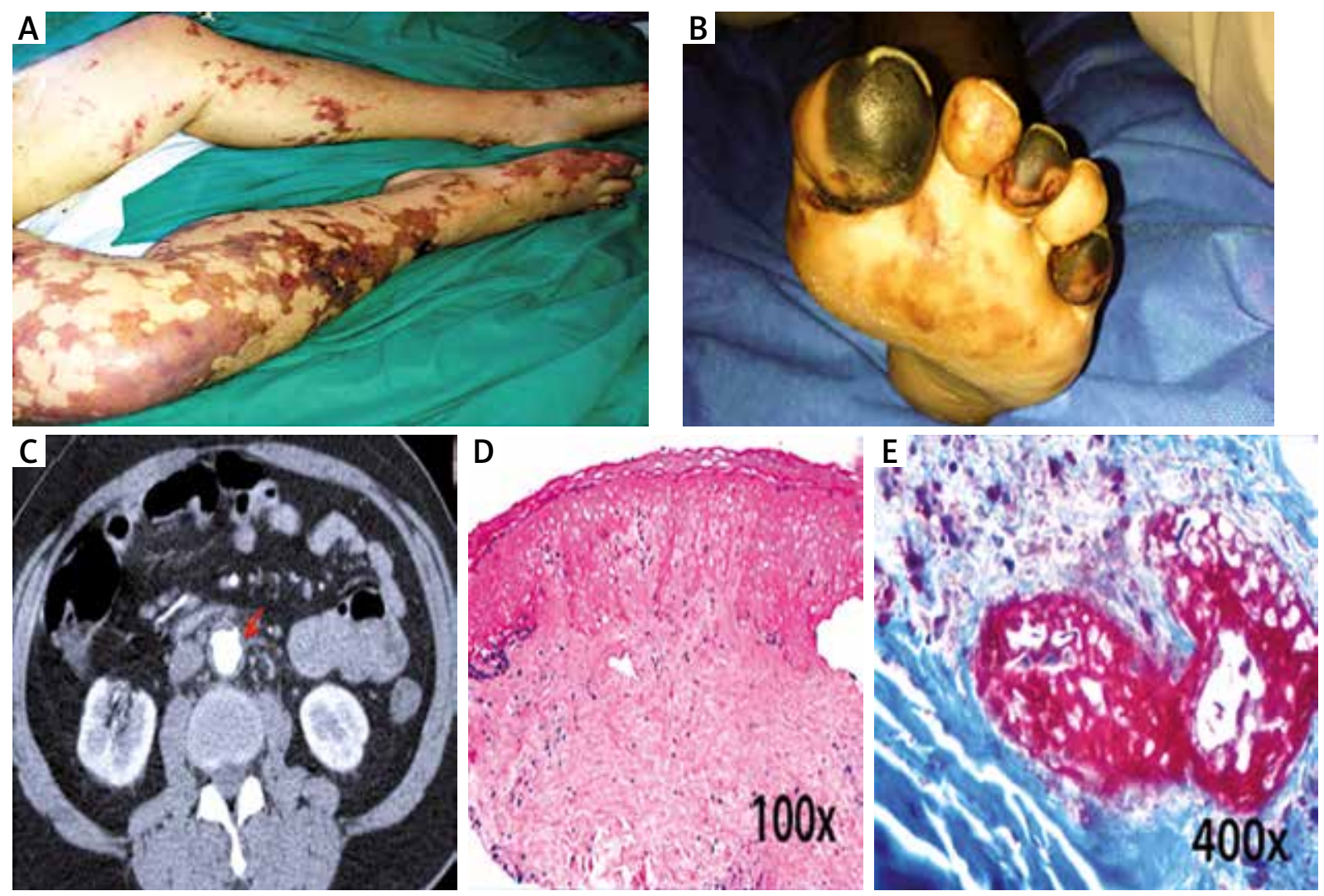

Figure 1. A - Extensive violaceous patches in a net-like pattern with ulcers and blisters on his lower extremities. B - Gangrene toes. C - Thorax-abdomen CT scan with contrast. The arrow indicates a complicated atherosclerotic plaque with thrombosis in the descending aorta. D - Full thickness epidermal necrosis with reticular dermis showing vascular occlusion by intraluminal amorphous eosinophilic thrombi. HE, 100x. E - Dermal blood capillary vessel with intraluminal hyaline thrombi with full occlusion of the lumen. There is no inflammation or vasculitis. Masson's trichrome stain, 400x

Livedo reticularis is a key sign for suspecting systemic microembolism in a patient with a history of thrombolytic therapy. It is an under-diagnosed clinical entity which is often related with increased in-hospital mortality. Since treatment options are limited without proven efficacy, increased awareness by the clinicians is needed and the diagnosis to be suspected with the triad of livedo reticularis, renal failure and eosinophilia. Therefore, we highlight this important cutaneous sign to be kept in mind especially in patients with recent thrombolysis.

\section{Acknowledgments}

This article was supported by the University of La Frontera (DI15-0097).

\section{Conflict of interest}

The authors declare no conflict of interest.

\section{References}

1. Mooney T, Joseph P. Purple toes syndrome following stroke thrombolysis and warfarin therapy. Intern Med J 2014; 44: 107-8.

2. Konstantinou DM, Chatzizisis YS, Farmakis G, et al. Cholesterol embolization syndrome following thrombolysis during acute myocardial infarction. Herz 2012; 37: 231-3.

3. García JM, Sobrino JM, Elizalde J, et al. Benign atheroembolic syndrome secondary to systemic fibrinolysis with streptokinase. Rev Esp Cardiol 1999; 52: 454-6.

4. Modi KS, Rao VK. Atheroembolic renal disease. J Am Soc Nephrol 2001; 12: 1781-7.

5. Moll S, Huffman J. Cholesterol emboli associated with warfarin treatment. Am J Hematol 2004; 77: 194-5. 